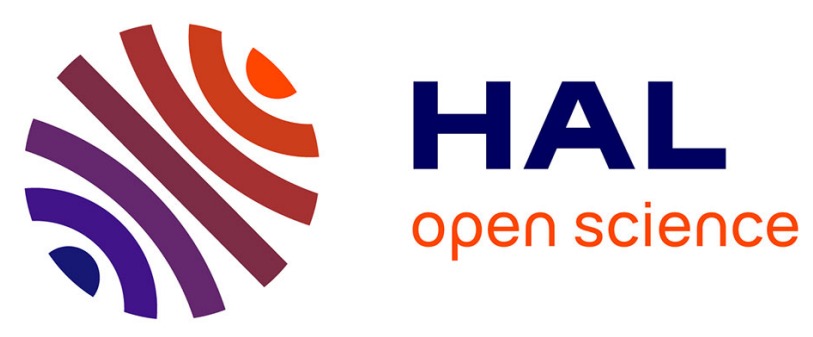

\title{
Charge Transfer and Tunable Ambipolar Effect Induced by Assembly of Cu (II) Binuclear Complexes on Carbon Nanotube Field Effect Transistor Devices
}

Gurvan Magadur, Jean-sébastien Lauret, Gaëlle Charron, Fatima Bouanis, Evgeny Norman, Vincent Huc, Costel Sorin Cojocaru, S. Goì mez-Coca, E. Ruiz, Talal Mallah

\section{To cite this version:}

Gurvan Magadur, Jean-sébastien Lauret, Gaëlle Charron, Fatima Bouanis, Evgeny Norman, et al.. Charge Transfer and Tunable Ambipolar Effect Induced by Assembly of Cu (II) Binuclear Complexes on Carbon Nanotube Field Effect Transistor Devices. Journal of the American Chemical Society, 2012, 134 (18), pp.7896-7901. 10.1021/ja301362r . hal-00793937

\section{HAL Id: hal-00793937 https://hal.science/hal-00793937}

Submitted on 10 Apr 2013

HAL is a multi-disciplinary open access archive for the deposit and dissemination of scientific research documents, whether they are published or not. The documents may come from teaching and research institutions in France or abroad, or from public or private research centers.
L'archive ouverte pluridisciplinaire HAL, est destinée au dépôt et à la diffusion de documents scientifiques de niveau recherche, publiés ou non, émanant des établissements d'enseignement et de recherche français ou étrangers, des laboratoires publics ou privés. 


\title{
Journal of the American Chemical Society
}

\author{
2012, volume $134 / 18$, pages $7896-7901$
}

dx.doi.org/10.1021/ja301362r

\section{Charge Transfer and Tunable Ambipolar Effect Induced by Assembly of $\mathrm{Cu}$ (II) Binuclear Complexes on Carbon Nanotube Field Effect Transistor Devices}

\author{
Gurvan Magadur,* Jean-Sebastien Lauret,\# Gaelle Charron,* Fatima Bouanis, ${ }^{*}, \S$ Evgeny Norman,§ \\ Vincent Huc,* Costel-Sorin Cojocaru,§ Silvia Gomez-Coca, $a$ Eliseo Ruiz, $₫$ and Talal Mallah,* \\ *Institut de Chimie Moleculaire et des Materiaux d'Orsay, Université de Paris Sud 11, CNRS, Bat. 420, 15 rue Georges Clemenceau, \\ 91405 Orsay Cedex, France
}

\#Laboratoire de Photonique Quantique et Moleculaire, 61 Avenue du President Wilson, 94230 Cachan, France

§Laboratoire de Physique des Interfaces et Couches Minces, Ecole Polytechnique, F-91128 Palaiseau, France

\begin{abstract}
œDepartament de Química Inorganica and Institut de Recerca de Química Teorica i Computacional, Universitat de Barcelona, Diagonal 645, 08028 Barcelona, Spain
\end{abstract}

\begin{abstract}
Assembly of paramagnetic Cu2 complexes with a Schiff base scaffold possessing extended electron delocalization together with a quasi-planar structure onto carbon nanotubes induces a diameter-selective charge transfer from the complex to the nanotubes leading to an interestingly large and tunable ambipolar effect. We used complementary techniques such as electron paramagnetic resonance, absorption spectroscopy, and photoluminescence to ensure the success of the assembly process and the integrity of the complex in the nanohybrid. We carried out density functional theory type calculations to rationalize the experimental results, evidencing the selective enhanced interaction of the metal complexes with one type of nanotube.
\end{abstract}

\section{INTRODUCTION}

Single-wall carbon nanotubes (SWNTs) have been at the forefront in the field of molecular electronics, particularly for design of smart devices such as ultrasensitive probes or field- effect transistors (FET).1-4 Combining the unique electronic and optic properties of SWNTs in FETs can even lead to new and guarantee modifications of the carbon nanotube field effect transistor (CNFET) properties.17Molecular complexes built using a Schiff base scaffold, well known for their optical, magnetic, and conducting properties, possess the desired electronic structure and structural features to be considered as excellent candidates to form hybrids with and very efficient types of photodetectors.5-7 Device properties new synergetic behavior. To date, the properties of such can be tuned and optimized by the assembly of different molecules on the nanotubes.8-14 Recently, SWNTs were used to build a magnetic sensor that may potentially detect the magnetic moment of a single molecule.15,16 Another objective is to take advantage of the interaction via charge transfer, for example, between the assembled molecules and the nanotube to tune the transport properties of the device. This is true not only for pure organic molecules but also for paramagnetic complexes. The key point is to optimize the interaction between the assembled molecules containing the paramagnetic ion and the nanotubes without altering in an irreversible manner the electronic properties of the nanotubes. Grafting complexes through covalent bonds might introduce complexes 
interacting noncovalently with carbon nanotube- based electronic systems are still to be investigated. A vanadium Schiff base complex was already covalently grafted on multiwall carbon nanotubes in order to elaborate modified electrodes with enhanced electrochemical response for reductive electro- catalysis.22 Herein we report the noncovalent assembly of the binuclear complex [Cu2bisalophen] (noted 1 and depicted in Figure 1) on SWNTs. We demonstrate that the grafting process leads to a tuning of the CNFET device properties due to diameter-selective charge transfer between the complex and the nanotube and to the appearance of a marked tunable ambipolar behavior was chosen because its extended $\pi$ system is expected to undergo strong $\pi-\pi$ interactions with the walls of the electron-scattering centers that limit the performance and properties of the device, while noncovalent $\pi$ stacking interactions are more likely to preserve the SWNT conductance nanotubes. Synthesis of the organic ligands was already reported.23 1 is obtained as red crystals with an excellent yield (85\%) by addition of $\mathrm{Cu}(\mathrm{OAc}) 2$ (see Supporting Information) to a solution of the organic ligand. The complex is very soluble in dichloromethane (DCM) and dichloroethane (DCE). To ensure the efficiency of the grafting process and check the integrity of the complex we used complementary and independent spectroscopybased characterization techniques. Since those techniques can directly access molecules and carbon nanotubes characteristic transitions, which are highly sensitive to environment modifications, electron paramagnetic resonance spectroscopy (EPR), optical absorption spectroscopy, and photoluminescence spectroscopy measurements were performed on different samples.

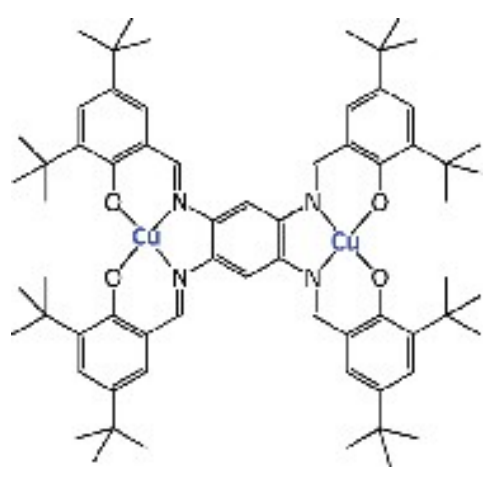

Figure 1. Schematic view of [Cu2bisalophen] 1.

\section{RESULTS AND DISCUSSION}

\subsection{Preparation and Preliminary Characterization of the Cu2/SWNT Adduct.}

Pristine HiPCO SWNTs were purified using a procedure derived from that reported by Chiang et al.24,25 The purity of the obtained SWNT was proven among other techniques by the absence of the D band in the Raman spectra (Supporting Information). Grafting of 1 was achieved by sonication of a suspension of the purified nanotubes in a solution of dichloromethane and dimethylfor- mamide (1:1) containing the complexes. The suspension was then filtered on a polytetrafluoroethane (PTFE) membrane and rinsed thoroughly several times with dichloromethane to remove all nongrafted complexes. A gray fine powder of the adduct (noted $1 @$ SWNT) was obtained and further analyzed. It is important to note here that a solution of the complex without the nanotubes was submitted to the same sonication process and then analyzed with EPR and electronic spectros- copy, which ensured the integrity of the molecules. EPR spectra were recorded for 1 in a solution of $\mathrm{CH}_{2} \mathrm{Cl}_{2}$ and DMF (1:1), for the purified SWNT, and for 1@SWNT powder (Figure 2). EPR spectra of 1 correspond to what is expected for a binuclear $\mathrm{Cu}$ (II) complex with axial local symmetry of the Cu sites.23 The resonance spectrum of $1 @$ SWNT is found at the same magnetic field value $(\sim 3100 \mathrm{G})$ as for 1 , indicating the presence of the binuclear complex on the nanotubes walls despite the abundant rinsing process.26 This is the first evidence of the success of the noncovalent assembly of the complex on the nanotubes. The sharp band around $3340 \mathrm{G}$ is attributed to the pure carbon nanotubes (Figure 2). 


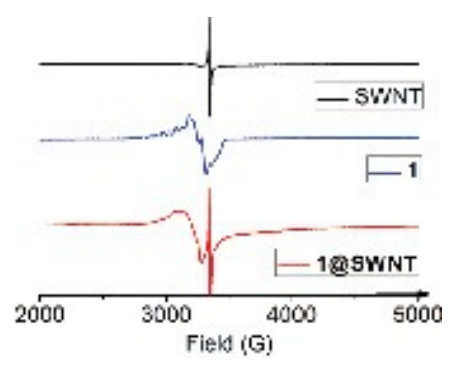

Figure 2. Comparison of EPR spectra of purified nanotubes (powder) (black curve), free 1 in $\mathrm{CH}_{2} \mathrm{Cl}_{2} / \mathrm{DMF}$ (1:1) solution (blue curve), and 1@SWNT (powder) obtained after filtration and extensive washing (red curve).

\subsection{Electronic Spectroscopy and Photoluminescence Studies.}

Along with this first characterization, an independent method used by Roquelet et al.27 was used to graft 1 onto the SWNT. This method, different from the previous one, ensures the presence of isolated nanotubes in solution, which is compulsory to investigate the photoluminescence properties of the adducts. The raw HiPCo nanotubes were dispersed in a solution of $\mathrm{pH} 8$ buffer plus $2 \mathrm{wt} \%$ sodium cholate (surfactant) using an ultrasound tip (see Supporting Information). The complex, dissolved in $\mathrm{CH}_{2} \mathrm{Cl}_{2}$, was then added to this solution and submitted again to ultrasound, leading to a biphasic system with formation of micelles in the aqueous phase. During evaporation of the organic phase, the molecular complexes insoluble in water are expected to diffuse inside the micelles, enabling their assembly on the individual SWNT coated by the surfactant and present in solution. The aqueous phase is then analyzed with electronic (UV-vis, nearinfrared) and photoluminescence spectroscopies.According to analogous measurements,12,14,27,28 the UV-vis spectrum of 1 is notably modified after the grafting process; the metal to ligand charge transfer (MLCT) band (450-600 nm) is red shifted by $17 \mathrm{~nm}$ (Figure 3), confirming the interaction between the two species. Since this shift is not observed for 1 in solution in the presence of surfactant (Figure 3top, green curve) and is not increasing for larger complex concentrations (see Supporting Information) it cannot be attributed to an interaction between the molecules and the surfactant or to molecules aggregation. Since the MLCT bands are the only ones affected, this shift may be attributed to a slight geometry modification or to a weak charge transfer process that affects the energy of the metal-centered molecular orbitals upon interaction with the nanotube. Another important feature is observed in the electronic spectra of the nanotube bands. A solvatochromic red shift attributed to coating of the nanotube by the molecules can also be seen for the SWNT (n,m)-type absorption bands between 900 and $1200 \mathrm{~nm}$ (Figure 3, bottom), confirming their interaction with 1. The impact of the molecules on the nanotube absorption properties is expected to be smaller than that of a nanotube on the molecules' properties since the SWNTs are mesoscopic objects and can alter several complexes at a time. The red shift is therefore weaker and ranges from 0 to $5 \mathrm{~nm}$. From the absorption spectra this shift does not seem to occur for all $(n, m)$ types of nanotubes. We have thus investigated the nanotubes dispersion photoluminescence spectra. The results confirmed the red shift for only some $(n, m)$ nanotube types (Figure 4). Using maps of photoluminescence excitation (PLE), which gave us the relative ratio of the $(n, m)$ types of SWNT and an experimental Kataura plot,29 we attributed each emission band of the photo- luminescence spectrum (Figure 4 ) to a $(n, m)$ type. The attribution is made for the most abundant type even though the band attributed to $(7,6)$ contains a contribution from the $(9,4)$ and $(8,4)$ types as well. The most affected type of nanotube within the range of excitation of $532 \mathrm{~nm}$ is the $(7,6)$ one $\left(\lambda_{11}=1130 \mathrm{~nm}\right)$. This band undergoes a shift of 5 $\mathrm{nm}$ upon assembly of 1 without a decrease of its intensity. While the (8,1)-type band has a shift of $3.5 \mathrm{~nm}$, the $(10,2)$ type band of 2 $\mathrm{nm}$ and the $(8,6)$ type band are not shifted at all; the intensity of these latter bands decreases slightly. These modifications are attributed to a diameter-selective charge transfer, particularly for the $(7,6)$-type band, where only a shift is observed and no intensity decrease. Diameter selection cannot be excluded since the intensity of some types (the smallest) decreases without an energy shift. 

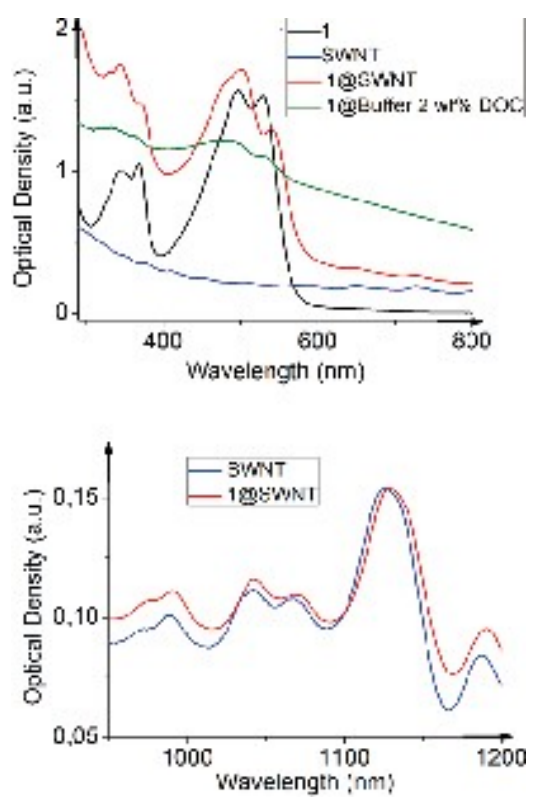

Figure 3. (Top) UV-vis spectra of 1 (black curve, MLCT bands between 400 and $600 \mathrm{~nm}$ ), 1 in pH 8 buffer with 2 wt \% sodium cholate (green curve), raw HiPCO SWNT in pH 8 buffer with 2 wt \% sodium cholate (blue curve), and 1 with SWNT in pH 8 buffer with 2 wt \% sodium cholate after evaporation of $\mathrm{CH}_{2} \mathrm{Cl}_{2}$ (red curve). (Bottom) Near-infrared spectra obtained with the same samples of raw HiPCO SWNT in pH 8 buffer with 2 wt \% sodium cholate (blue curve) and 1 with SWNT in $\mathrm{pH} 8$ buffer with 2 wt \% sodium cholate after evaporation of $\mathrm{CH}_{2} \mathrm{Cl}_{2}$ (red curve).
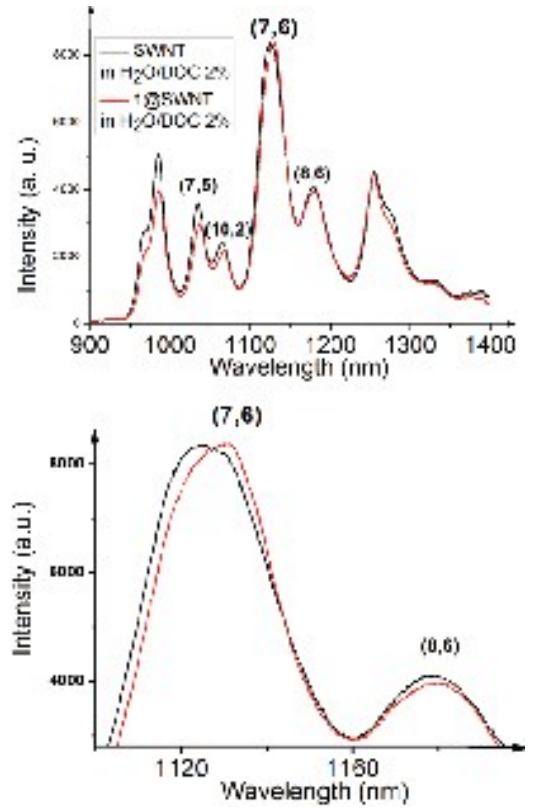

Figure 4. (Top) Photoluminescence spectra of raw SWNT (black curve) and 1@SWNT both in pH 8 buffer with 2 wt \% sodium cholate and $\mathrm{CH}_{2} \mathrm{Cl}_{2}$. (Bottom) Zoom on the $(7,6)$ band.

\subsection{Theoretical Calculations.}


In order to rationalize the molecule-nanotube interaction effects theoretical density functional calculations (DFT) were performed with the Siesta code30,31 (see Supporting Information) for the $(n, m)$ nanotube type that seems to be the most affected during the grafting process: the $(7,6)$. The more stable optimized geometry has a perpendicular orientation as shown in Figure 5 . Time-dependent DFT calculations based on this optimized structure were then performed to determine the vertical excitations of 132 before and after grafting.33 Without solvent the calculated excitations were found at 487 and $523 \mathrm{~nm}$ for 1 , which is in fair agreement with the experimental values (494 and $528 \mathrm{~nm}$, respectively). For $1 @ S W N T$, the calculations show a shift of the two bands to 520 and $568 \mathrm{~nm}$ for the interacting molecule to be compared to the experimental values of 507 and $547 \mathrm{~nm}$. Even though the calculations do not reproduce exactly the values of the absorption band energies, they reproduce the experimental red shift trend and confirm the origin of the change in energy of the absorption bands as being due to the interaction between the binuclear Cu2 complex and the nanotubes.

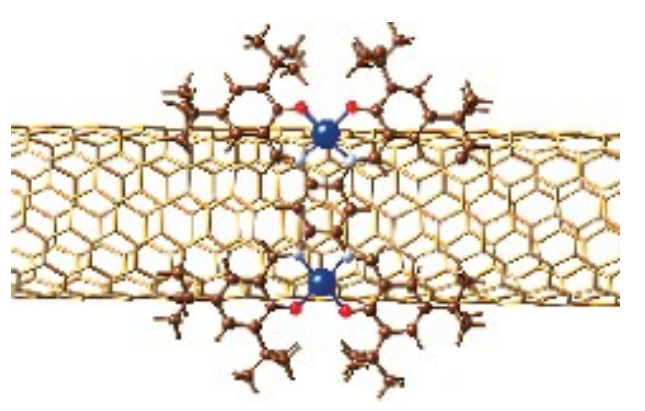

Figure 5. View of the optimized structure (perpendicular orientation for 1 grafted onto a $(7,6)$ nanotube from theoretical calculations). Blue, red, light blue, and brown spheres correspond to copper, oxygen, nitrogen, and carbon atoms, respectively, and hydrogen atoms are indicated as brown sticks.

\subsection{Transport Studies.}

The studies performed above demonstrate two important features: first, the binuclear complex adheres well onto the nanotubes as evidenced by EPR and, second, there is an electronic interaction between the paramagnetic complexes and the nanotubes as evidenced by the electronic and photoluminescence spectra and confirmed by theoretical calculations. In addition to this electronic interaction one may expect the presence of a charge transfer between the molecules and the nanotubes. Even though some techniques like Raman are suitable to study the charge transfer, investigation of the transport behavior may give more complete information about the electronic interaction occurring between the nanotubes and 1 and evidence other electronic phenomena, such as gate-dependent ones, not possible to account for otherwise. The transport studies were carried out using a CNTFET device in ambient air. The device was obtained following a new strategy based on self-assembled monolayers (SAMs), which has been described (see Supporting Information).34 Since the interaction was proved between 1 and HiPCO SWNT, the grafting results were thought to be identical or close to those existing with the HFCVD nanotubes used in these devices. The characteristics of the FET device were first investigated. Current $(\mathrm{ld})$ versus gate voltage $\left(\mathrm{V}_{\mathrm{g}}\right)$ curves show a p-type FET behavior (ON state at $\mathrm{V}_{\mathrm{g}}<0$ ) and no ambipolar effect, as often found for silica CNFET in ambient air,35-37 with a very good lon/loff ratio of four decades. Indeed, the ambipolar behavior is usually only observed when oxygen and water are removed by pumping, thus restoring the expected properties of the nanotubes that are intrinsic semiconductors. Hysteresis is observed as a result of charge injection from the CNT to the nearby region (Figure 6).38 


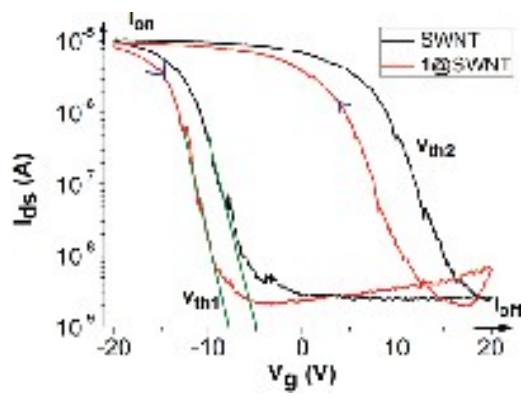

Figure 6. Ids vs $\bigvee_{\mathrm{g}}$ curves for a CNFET device before (black curve) and after grafting of 1 and rinsing with DCE process. Experimental starting gate potential is $-20 \mathrm{~V}$, and current evolution with $\mathrm{V}_{\mathrm{g}}$ is given by the blue arrows.

The hybrids were then produced by evaporation of a $5 \mu \mathrm{L}$ drop of a solution of 1 in DCE deposited on the CNTFET surface followed by a thorough DCE washing process of the device to remove any nongrafted molecules. Ids $V_{s} V_{g}$ measurements were then performed. The subthreshold swing $S_{w}=\left[d V_{g} / d \log (I)\right]$ (green slopes in Figure 6) remains unchanged upon grafting, indicating that treatment leaves the gate unaffected. However, the lon current decreases, implying that the complexes are interacting with the electron flow, producing scattering centers.1 While no appreciable variation is observed after repeated treatment of the FET with DCE alone (see Supporting Information), drastic changes can be observed on the $\mathrm{d}_{\mathrm{ds}} \mathrm{Vs} \mathrm{V}_{\mathrm{g}}$ hysteresis curves after the grafting operation (Figure 6). The ON/OFF threshold voltage $V_{\text {th } 1}$ decreases from -5 to $-8 \mathrm{~V}$ as 1 interacts with the nanotubes sidewalls, and an ambipolar characteristic appears for high $\mathrm{V}_{\mathrm{g}}$ values. These changes are attributed to charge trapping as well as charge transfer from the complexes to the SWNTs ( $\mathrm{V}_{\text {th1 }}$ decreases),39 evidencing a $n$-type doping on the nanotube, that is, a transfer of negative charges. For this gate sweep the device is first in the p-type ON state. Then holes in the nanotube vicinity are detrapping while the gate is swept toward positive values, until the OFF state is reached. Finally, enhanced by the grafting of 1 onto the SWNT, electrons start to be trapped in the nanotube vicinity while the device is slowly reaching its n-type ON state. This experiment is definitive proof of the occurrence of an electronic density transfer from the paramagnetic complexes to the SWNTs. In order to get a better understanding of the origin of the Cu2 complex/nanotube interaction, especially the phenomen- on responsible for the ambipolar behavior, several deposition washing cycles were performed (Figure 7, top).
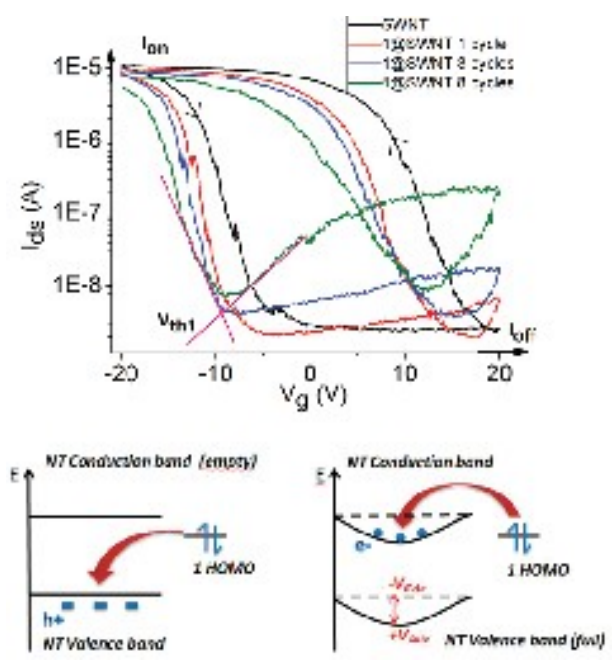

Figure 7. (Top) Ids vs $V_{\mathrm{g}}$ curves for a CNFET device before (black curve) and after a different number of grafting-rinsing cycles with 1. Experimental starting gate potential is $-20 \mathrm{~V}$, and evolution of the current is given by the black arrows on the black curve. (Bottom left) Electrons transfer from the complexes HOMO to the p-type SWNTs valence band. (Bottom right) As $V_{\text {Gate }}$ becomes positive, the bands 
are bending toward lower energies, allowing electron transfer from the HOMO of the complexes to the conduction band of the SWNTs. CNFET thus becomes n-type for positive $\mathrm{V}_{\text {Gate }}$ values, leading to an ambipolar characteristic.

As the number of cycles increases, $V_{\text {th1 }}$ decreases and the ambipolar effect becomes more and more pronounced. While the increased shift of the hysteresis curve can be attributed to increasing electron transfer from the complexes to the SWNTs as more and more molecules are grafted onto their sidewalls, the origin of the change in the magnitude of the ambipolar effect is not straightforward. As various external factors can affect the $I_{d s} v s V_{g}$ characteristic, several aspects must be checked to ensure that the more pronounced ambipolar phenomena is indeed related to the increase of the molecules' concentrations on the nanotube and not to other effects. Since the subthreshold swing is not affected by the grafting and since the rinsing process removes all nongrafted and

substrate-deposited molecules one can safely assume that the observed effects are not due to the molecules perturbing the environment of the nanotubes conducting channels. Moreover, since repeated treatments by DCE alone have no effect on the lds Vs $V_{g}$ characteristic (see Supporting Information) and since the n-type subthreshold (positive slope in Figure 7, top) swing increases with the number of cycles, the ambipolar effect cannot be due to external effects but only to the noncovalent interaction between the molecules and the nanotubes. To understand the intrinsic character of the ambipolar effect one needs to consider Figure 7 (bottom). As $V_{\text {Gate }}$ becomes positive, the conduction band of the nanotubes is bending toward lower energies, thus being able to receive electrons from the HOMO of the molecules. The electrons then become the main charge carriers, leading to an $\mathrm{n}$-type behavior. This interpretation is supported by the fact that the intersection of the two pink slopes (Figure 7bottom), indicative of the potential for which the conduction type starts to change, occurs around the same $\mathrm{V}_{\text {Gate }} \approx-10 \mathrm{~V}$ value for both the third and the eighth cycle. However, we do not exclude the fact that this effect is also due in part to a global shift of the characteristic due to the existing charge transfer process.37This reproducible, easy to generate, and tunable ambipolar character is of major interest since most logic circuits need to integrate both $\mathrm{n}$ - and $\mathrm{p}$-type conductors, and the presence of both kinds of charge carriers can lead to exciton recombination and thus photo- luminescence phenomena.

\section{CONCLUSION}

We have thus demonstrated that the Cu2bisalophen complexes can be assembled in a noncovalent manner on SWNTs elaborated by HiPCO and hot filament-assisted chemical vapor deposition techniques. The origin of the nanotubes seems to not affect the electronic interaction responsible for this assembly. However, electron transfer from the molecules to the nanotubes is only evidenced with the transport studies. Noncovalent grafting of metal transition complexes onto SWNTs in a CNFET channel leads to electron transfer from the molecules to the nanotube and generates a tunable ambipolar effect in ambient air conditions. This opens the possibility to design new kinds of nanohybrid circuits. Beyond this important result, new kinds of CMOS inverters or logic circuits may be realized as well as new powerful photocurrent devices by combining the evergrowing number of optic properties of inorganic complexes with promising optoelec- tronic ones of SWNTs. Furthermore, the mere presence of an electronic interaction between the conducting electrons of the nanotubes and the localized electrons of the paramagnetic complex may be used to tune the exchange coupling interaction of the binuclear paramagnetic species. In addition, a diameter- selective charge transfer between the complex and the nanotubes seems to occur, which will be the subject of further investigations. Schiff base binuclear complexes are particularly appealing since they are relatively easy to prepare. More importantly, they are very stable in solution, which is a requirement for elaboration of the modified CNFET. Their electronic and magnetic properties may be tuned by changing the nature of the metal ions and modifying the organic ligand itself adding donor or acceptor groups. Thus, they offer an attractive playground for tuning the electronic behavior of CNFET devices.

\section{* Supporting Information}

Synthetic procedure and full characterization of the Cu2 complex, procedure for purification of the nanotubes, spectra of the hybrids, procedure for fabrication and characterization of the CNFET, and methods used for DFT calculations. This material is available free of charge via the Internet at http:// pubs.acs.org. 
ACKNOWLEDGMENTS We thank the CNRS, the Universite Paris Sud 11, and the Ecole Polytechnique for financial support. The research described here has been also supported by Triangle de la Physique project SECOND, convention no. 2010-42T, and the ANR project MolNanoSpin: 08-NANO-P110-48.

\section{REFERENCES}

(1) Avouris, P.; Chen, Z. H.; Perebeinos, V. Nat. Nanotechnol. 2007, 2, 605.

(2) Besteman, K.; Lee, J. O.; Wiertz, F. G. M.; Heering, H. A.;Dekker, C. Nano Lett. 2003, 3, 727.

(3) Kong, J.; Franklin, N. R.; Zhou, C. W.; Chapline, M. G.; Peng, S.; Cho, K. J.; Dai, H. J. Science 2000, 287, 622.

(4) Tans, S. J.; Verschueren, A. R. M.; Dekker, C. Nature 1998, 393,49.

(5) Avouris, P.; Chen, J.; Freitag, M.; Perebeinos, V.; Tsang, J. C.Phys. Status Solidi B 2006, 243, 3197.

(6) Avouris, P.; Freitag, M.; Perebeinos, V. Nat. Photonics 2008, 2,341.

(7) Freitag, M.; Martin, Y.; Misewich, J. A.; Martel, R.; Avouris, P. H.Nano Lett. 2003, 3, 1067.

(8) Bogani, L.; Danieli, C.; Biavardi, E.; Bendiab, N.; Barra, A. L.; Dalcanale, E.; Wernsdorfer, W.; Cornia, A. Angew. Chem., Int. Ed.2009, $48,746$.

(9) Campidelli, S.; Le Ho, K. H.; Rivier, L.; Jousselme, B.; Jegou, P.;Filoramo, A. Chem. Commun. 2010, 46, 8731.

(10) Charron, G.; Giusti, A.; Mazerat, S.; Mialane, P.; Gloter, A.;Miserque, F.; Keita, B.; Nadjo, L.; Filoramo, A.; Riviere, E.; Wernsdorfer, W.; Huc, V.; Bourgoin, J. P.; Mallah, T. Nanoscale 2010, 2, 139.

(11) Giusti, A.; Charron, G.; Mazerat, S.; Compain, J. D.; Mialane, P.;Dolbecq, A.; Riviere, E.; Wernsdorfer, W.; Bibouni, R. N.; Keita, B.; Nadjo, L.; Filoramo, A.; Bourgoin, J. P.; Mallah, T. Angew. Chem., Int. Ed. 2009, 48, 4949.

(12) Magadur, G.; Lauret, J. S.; Alain-Rizzo, V.; Voisin, C.;Roussignol, P.; Deleporte, E.; Delaire, J. A. ChemPhysChem 2008, 9, 1250.

(13) van der Lelie, D.; Zhang, Y. B.; Kanungo, M.; Ho, A. J.;Freimuth, P.; Chen, M.; Khamis, S. M.; Datta, S. S.; Johnson, A. T. C.; Misewich, J. A.; Wong, S. S. Nano Lett. 2007, 7, 3086.

(14) Weisman, R. B.; Casey, J. P.; Bachilo, S. M. J. Mater. Chem. 2008, 18, 1510.

(15) Cleuziou, J. P.; Wernsdorfer, W.; Bouchiat, V.; Ondarcuhu, T.;Monthioux, M. Nat. Nanotechnol. 2006, 1, 53.

(16) Urdampilleta, M.; Klyatskaya, S.; Cleuziou, J. P.; Ruben, M.; Wernsdorfer, W. Nat. Mater. 2011, 10, 502.

(17) Park, H.; Zhao, J.; Lu, J. P. Nano Lett. 2006, 6, 916.

(18) Chou, K. C.; Pietrangelo, A.; Sih, B. C.; Boden, B. N.; Wang, Z. W.; Li, Q. F.; MacLachlan, M. J.; Wolf, M. O. Adv. Mater. 2008, $20,2280$.

(19) Demartin, F.; Rigamonti, L.; Forni, A.; Righetto, S.; Pasini, A.Inorg. Chem. 2006, 45, 10976.

(20) MacLachlan, M. J.; Leung, A. C. W.; Hui, J. K. H.; Chong, J. H. Dalton Trans. 2009, 5199.

(21) Zou, D. C.; Qu, L.; Wang, D.; Zhong, C.; Zou, Y. J.; Li, J.; Qin, J. G. Synth. Met. 2010, 160, 2299.

(22) Salimi, A.; Mamkhezri, H.; Mahebbi, S. Electrochem. Commun. 2006, 8, 688.

(23) Kleij, A. W.; Kuil, M.; Tooke, D. M.; Lutz, M.; Spek, A. L.; Reek, J. N. H. Chem.?Eur. J. 2005, 11, 4743.

(24) Charron, G.; Mazerat, S.; Erdogan, M.; Gloter, A.; Filoramo, A.; Cambedouzou, J.; Launois, P.; Riviere, E.; Wernsdorfer, W.; Bourgoin, J. P.; Mallah, T. New J. Chem. 2009, 33, 1211.

(25) Chiang, I. W.; Brinson, B. E.; Huang, A. Y.; Willis, P. A.; Bronikowski, M. J.; Margrave, J. L.; Smalley, R. E.; Hauge, R. H. J. Phys. Chem. B 2001, $105,8297$.

(26) However, the spectrum does not show the hyperfine structure of the isolated binuclear complex may be because of the presence of an interaction between the electronic density of the $\mathrm{Cu}(\mathrm{II})$ with that of the nanotubes. This aspect merits being investigated but is out of the scope of this paper.

(27) Roquelet, C.; Lauret, J. S.; Alain-Rizzo, V.; Voisin, C.; Fleurier, R.; Delarue, M.; Garrot, D.; Loiseau, A.; Roussignol, P.; Delaire, J. A.; Deleporte, E. ChemPhysChem 2010, 11, 1667.

(28) Rao, C. N. R.; Voggu, R.; Rout, C. S.; Franklin, A. D.; Fisher, T. S. J. Phys. Chem. C 2008, 112, 13053.

(29) Weisman, R. B.; Bachilo, S. M. Nano Lett. 2003, 3, 1235.

(30) Dion, M.; Rydberg, H.; Schroder, E.; Langreth, D. C.; Lundqvist, B. I. Phys. Rev. Lett. 2004, 92, 246401.

(31) Soler, J. M.; Artacho, E.; Gale, J. D.; Garcia, A.; Junquera, J.; Ordejon, P.; Sanchez-Portal, D. J. Phys. Condens. Mattter 2002, $14,2745$.

(32) Bauernschmitt, R.; Ahlrichs, R. Chem. Phys. Lett. 1996, 256, 454. (33) Adamo, C.; Barone, V. J. Chem. Phys. 1999, $110,6158$.

(34) Bouanis, F. Z.; Baraton, L.; Huc, V.; Pribat, D.; S., C. C. Thin Solid Films 2011, 519, 4594. 
(35) Derycke, V.; Martel, R.; Appenzeller, J.; Avouris, P. Appl. Phys. Lett. 2002, 80, 2773.

(36) Derycke, V.; Martel, R.; Appenzeller, J.; Avouris, P. Nano Lett. 2001, 1, 453.

(37) Aguirre, C. M.; Levesque, P. L.; Paillet, M.; Lapointe, F.; St- Antoine, B. C.; Desjardins, P.; Martel, R. Adv. Mater. $2009,21,3087$.

(38) Kim, W.; Javey, A.; Vermesh, O.; Wang, O.; Li, Y. M.; Dai, H. J. Nano Lett. 2003, 3, 193.

(39) Star, A.; Han, T. R.; Gabriel, J. C. P.; Bradley, K.; Gruner, G. Nano Lett. 2003, 3, 1421. 